\title{
A Nonlinear Drift Memristor Model with a Modified Biolek Window Function and Activation Threshold
}

\author{
Valeri Mladenov and Stoyan Kirilov * \\ Department of Theoretical Electrical Engineering, Technical University Sofia, Faculty of Automatics, 1000 Sofia, \\ 8 St. Kl. Ohridski Blvd, Bulgaria; valerim@tu-sofia.bg \\ * Correspondence: s_kirilov@tu-sofia.bg; Tel.: 00359-2-965-33-19
}

Received: 28 August 2017; Accepted: 29 September 2017; Published: 3 October 2017

\begin{abstract}
The main idea of the present research is to propose a new memristor model with a highly nonlinear ionic drift suitable for computer simulations of titanium dioxide memristors for a large region of memristor voltages. For this purpose, a combination of the original Biolek window function and a weighted sinusoidal window function is applied. The new memristor model is based both on the Generalized Boundary Condition Memristor (GBCM) Model and on the Biolek model, but it has an improved property - an increased extent of nonlinearity of the ionic drift due to the additional weighted sinusoidal window function. The modified memristor model proposed here is compared with the Pickett memristor model, which is used here as a reference model. After that, the modified Biolek model is adjusted so that its basic relationships are made almost identical with these of the Pickett model. After several simulations of our new model, it is established that its behavior is similar to the realistic Pickett model but it operates without convergence problems and due to this, it is also appropriate for computer simulations. The modified memristor model proposed here is also compared with the Joglekar memristor model and several advantages of the new model are established.
\end{abstract}

Keywords: memristor; nonlinear ionic drift; modified Biolek window function; sinusoidal weighted window function; sensitivity threshold

\section{Introduction}

Background and concept. The memristor (abbreviated from memory + resistor) is the fourth electrical passive two-terminal element alongside with the resistor, inductor, and capacitor $[1,2]$. The memristor is a nonlinear electrical element [1]. It directly relates the electric charge $q$ and the magnetic flux linkage $\Psi$ [1,2]. Leon Chua forecast the memristor element in 1971 in accordance to symmetry considerations and the relationships between the four basic electrical quantities-voltage, current, charge, and magnetic flux [1]. The memristor element has the property for memorizing the amount of charge passed through its intersection (in given limits depending on the geometrical dimensions of the element) and its respective resistance (conductance), which is proportional to the electric charge accumulated in the memristor when the electric sources are switched off [2]. The Weber-Coulomb relationship of the memristor is a nonlinear single-valued curve, while its respective current-voltage relationship is a pinched-hysteresis loop, which shape and area depend on the amplitude and on the frequency of the applied voltage [2]. Due to the effect of memorizing the resistance of the memristor after switching off the electrical sources, it could be used as a non-volatile memory element [2]. Its physical prototype made by titanium dioxide [2-4] has physical dimensions in the nano scale range and its power consumption is several times lower than the existing flash memories [2]. These facts are good preconditions for the future applications of the memristor matrices in computer memories [2]. The memristor element is a good candidate for application 
in the neuromorphic engineering, in the synapses in the artificial neural networks, in the analog and digital electronics as well [2].

The first physical prototype of the memristor element predicted by L. Chua in 1971 [1] was invented in the HP research labs by Stanley Williams research team in 2008 [2]. After this flash, many scientific papers associated with memristors and memristive devices have been published and several basic memristor models have been proposed [2-10]. Each of the basic memristor models-the linear drift model proposed by Strukov and Williams [2] and the nonlinear drift models made by Joglekar [3], Pickett [4,5], and Biolek [6]—is appropriate for specific electrical modes for the operation of the memristor elements. The linear ionic drift model [2] is used for low memristor voltages and the nonlinear drift models of Biolek and Joglekar are able to represent the memristor nonlinear drift behavior for high voltages when the memristor element has highly nonlinear performance $[3,6]$. The Pickett memristor model $[4,5]$ is based on laboratory experiments and measurements, and on the current flow through a tunnel barrier. It has the highest accuracy and sometimes it is used as a reference model $[4,5]$, but it is very complicated and it is not always appropriate for computer simulations due to convergence problems. The Boundary Condition Memristor (BCM) Model is with linear dopant drift and switch-based window function for representation the boundary effects [7]. It is able to represent both the soft-switching and hard-switching memristor modes [7]. The Generalized BCM model (GBCM) created by Ascoli, Corinto, and Tetzlaff, is almost identical with the BCM model but the difference is the use of activation threshold not only for the boundaries but also for every value of the state variable [8]. The lack of a realistic versatile memristor model suitable for simulations, having a nonlinear dopant drift and accuracy near to the Pickett model was the motivation of the present research. The new model proposed in this paper is based both on Biolek and on the GBCM models but it uses a combination of a Biolek window function and an additional weighted sinusoidal window function for increasing the nonlinearity of the ionic drift. The new ideas of the memristor model proposed here are that the weight coefficient in front of the additional sinusoidal window function component and the respective extent of nonlinearity of the memristor dopant drift could be changed and adjusted till the basic relationship of the memristor are almost near to these obtained by the Pickett model for the same conditions. If the activation threshold and the coefficient in front of the weighted sinusoidal window function are equal to zero the original Biolek model is obtained. The Biolek memristor model is a special case of the modified Biolek model. The boundary effects observable for hard-switching mode are represented using the switch-based algorithm of the GBCM model. After comparison with the Pickett memristor model the modified Biolek model was adjusted so its current-voltage and the Weber-Coulomb relationships were obtained similar to the Pickett reference model. The precision of the modified Biolek model is near to the accuracy of the Pickett memristor model but the computer tests confirmed that the modified Biolek model does not have convergence problems and it is appropriate for computer simulations of memristors and memristive circuits.

In Section 2, the description of the titanium-dioxide memristor element according to the Pickett Tunnel Barrier model is presented and the main relationships are obtained. The basic ideas and the major relationships of the modified Biolek model are shown in Section 3. The pseudo-code based algorithm of the modified Biolek model for computer simulations is presented in Section 4 . The comparison with the Pickett memristor model, the adjusting process of the modified Biolek model, and the basic results are presented and discussed in Section 5. A comparison of the model proposed here with Joglekar memristor model is presented and discussed in Section 6. The concluding remarks are presented in Section 7.

\section{A Brief Description of the Memristor According to the Pickett Tunnel Barrier Model}

The structure of a memristor cell according to the Pickett's model [5] is shown in Figure 1. The electrodes are made of platinum and the insulating layer is made of pure titanium dioxide. The conducting channel in the memristor cell is formed by a thin layer of doped with oxygen vacancies 
titanium dioxide material. There is also a thin tunnel barrier with a length of $w$. The resistance of the conducting layer is approximately equal to $R s=215 \Omega[4,5]$.

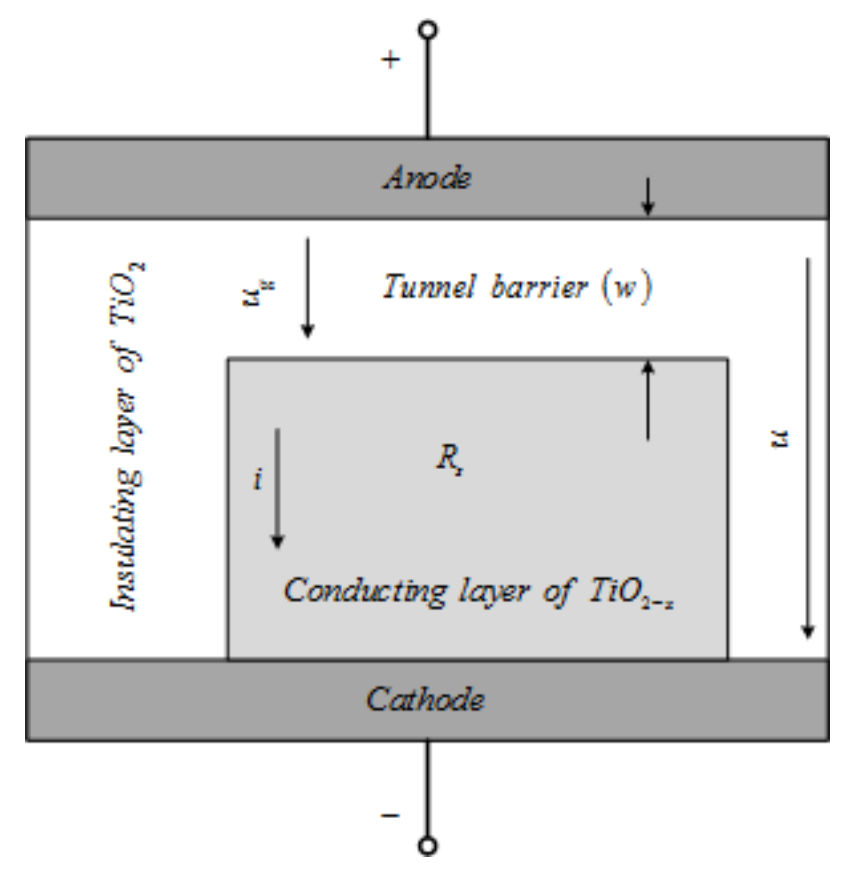

Figure 1. Memristor structure according to the Pickett model.

When the memristor is switched in open state $(i>0)$, the differential equation has to be expressed with (1) [5]. Formulas (2)-(11) are also according to [5].

$$
\frac{d w}{d t}=f_{o f f} \sinh \left(\frac{i}{i_{o f f}}\right) \exp \left[-\exp \left(\frac{w-a_{o f f}}{w_{c}}-\frac{|i|}{b}\right)-\frac{w}{w_{c}}\right]
$$

The parameters used in (1) and their corresponding values [5] are given in Table 1.

Table 1. Quantities for open switched memristor.

\begin{tabular}{cccccc}
\hline Quantity & $f_{\text {off }}$ & $\boldsymbol{i}_{\text {off }}$ & $\boldsymbol{a}_{\text {off }}$ & $\boldsymbol{b}$ & $\boldsymbol{w}_{\boldsymbol{c}}$ \\
\hline Dimension & $\mu \mathrm{m} / \mathrm{s}$ & $\mu \mathrm{A}$ & $\mathrm{nm}$ & $\mu \mathrm{A}$ & $\mathrm{pm}$ \\
Value & 3.5 & 115 & 1.20 & 500 & 107 \\
\hline
\end{tabular}

When the memristor is switched in closed state $(i<0)$ [5], the differential equation is:

$$
\frac{d w}{d t}=f_{o n} \sinh \left(\frac{i}{i_{o n}}\right) \exp \left[-\exp \left(\frac{w-a_{o n}}{w_{c}}-\frac{|i|}{b}\right)-\frac{w}{w_{c}}\right]
$$

The parameters and their values [5] are given in Table 2.

Table 2. Quantities for closed switched memristor.

\begin{tabular}{cccccc}
\hline Quantity & $\boldsymbol{f}_{\boldsymbol{o n}}$ & $\boldsymbol{i}_{\boldsymbol{o n}}$ & $\boldsymbol{a}_{\boldsymbol{o n}}$ & $\boldsymbol{b}$ & $\boldsymbol{w}_{\boldsymbol{c}}$ \\
\hline Dimension & $\mu \mathrm{m} / \mathrm{s}$ & $\mu \mathrm{A}$ & $\mathrm{nm}$ & $\mu \mathrm{A}$ & $\mathrm{pm}$ \\
Value & 40 & 8.9 & 1.80 & 500 & 107 \\
\hline
\end{tabular}


The current flowing through the tunnel barrier of the memristor element [5] is:

$$
i=\frac{j_{0} A}{\Delta w^{2}}\left[\Phi_{1} \exp \left(-B \sqrt{\Phi_{1}}\right)-\left(\Phi_{1}+\left|u_{g}\right|\right) \exp \left(-B \sqrt{\Phi_{1}}+\left|u_{g}\right|\right)\right]
$$

where $u_{g}$ is the voltage drop over the tunnel barrier [5]. This voltage drop could be expressed using the Kirchhoff's Voltage Law (KVL) and the voltage $u$ over the memristor element [5,11]:

$$
u_{g}=u-i R_{s}
$$

The constant $j_{0}[5]$ could be expressed as follows:

$$
j_{0}=\frac{e}{2 \pi h}=\frac{1.6 \cdot 10^{-19}}{2 \cdot 3.14 \cdot 6.63 \cdot 10^{-34}}=3.84 \cdot 10^{13},[C /(J \cdot s)]
$$

where $e$ is the elementary charge of the electron, $h$ is the Planck's constant.

The area of the tunnel junction [5] is equal to $A=10^{4} \mathrm{~nm}^{2}$.

The deviation of the length of the tunnel junction [5] is:

$$
\Delta w=w_{2}-w_{1}
$$

The second term of (6) [5] is:

$$
w_{1}=1.2 \frac{\lambda w}{\Phi_{0}}, n m^{2}
$$

where $\Phi_{0}=0.95 \mathrm{~V}$ is the height of the potential barrier. The quantity $\lambda$ [5] from Equation (7) is expressed as follows:

$$
\lambda=\frac{e \ln 2}{8 \pi k \varepsilon_{0} w \cdot 10^{-9}}, \quad V
$$

where $k=5$ is the permittivity of the titanium dioxide material, $\varepsilon_{0}=8.85 \cdot 10^{-12} \mathrm{~F} / \mathrm{m} \mathrm{[5]}$ is the absolute permittivity of the vacuum space.

After transformation of (7) it is obtained that $\mathrm{w}_{1}=0.126 \mathrm{~nm}$. Using (6) and according to [5] the following Equation (9) is derived:

$$
w_{2}=w_{1}+w\left(1-\frac{9.2 \cdot \lambda}{3 \Phi_{0}+4 \lambda-2\left|u_{g}\right|}\right), n m
$$

The quantity $B[5]$ is expressed as follows:

$$
B=\frac{4 \pi \Delta w \cdot 10^{-9} \sqrt{2 m e}}{h}, V^{-\frac{1}{2}}
$$

where $m$ is the electron weight. The quantity $\Phi_{1}[5]$ is:

$$
\Phi_{1}=\Phi_{0}-\left|u_{g}\right| \frac{w_{1}+w_{2}}{w}-\frac{1.15 \cdot \lambda w}{\Delta w} \ln \left[\frac{w_{2}\left(w-w_{1}\right)}{w_{1}\left(w-w_{2}\right)}\right], \quad V
$$

The Pickett memristor model [5] is fully described with Equations (1)-(11). A Simulation Program with Integrated Circuit Emphasis (SPICE) code of Pickett model [5] is created using (1)-(11) and it is used for simulation of the memristor element.

The memristor circuit under test is presented in Figure 2. It is created of a sinusoidal voltage source, a series resistor with very small resistance $\left(R_{1}=1 \Omega\right)$, a difference devices, and integrators. The magnetic flux linkage is obtained as a time integral of the voltage drop across the memristor, and the electric charge is the time integral of the memristor current and, respectively, the voltage drop across the resistor $R_{1}$ [11]. 


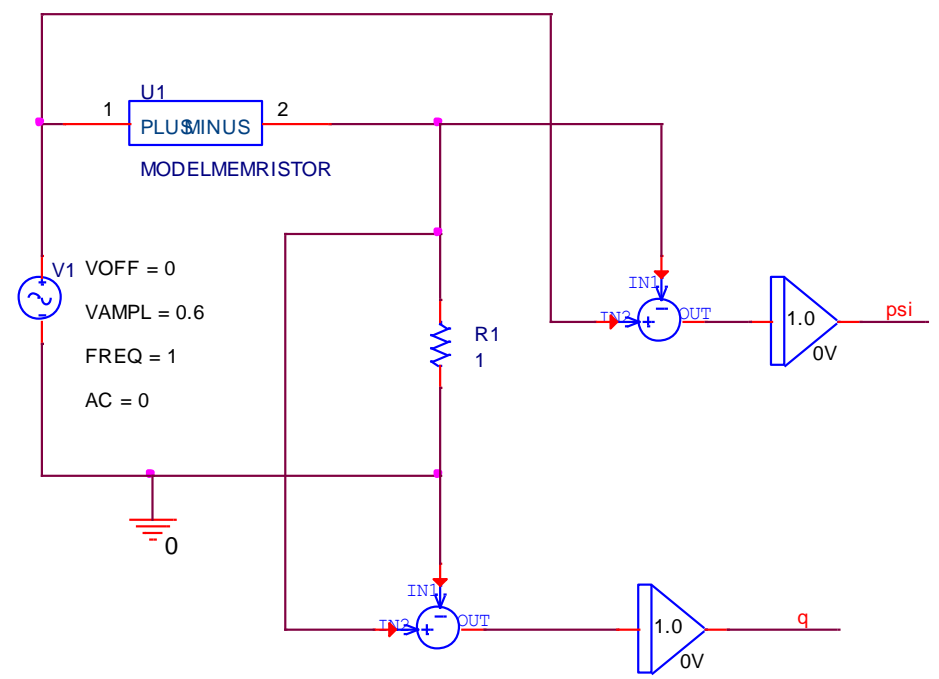

Figure 2. Memristor circuit for simulation in Simulation Program with Integrated Circuit Emphasis (SPICE) environment.

Figure 3 was the Weber-Coulomb relationship according to Pickett model and the I-V relationship of the memristor element according to Pickett model. The sinusoidal voltage signal generated by the voltage source and used for the computer simulation is: $u(t)=0.6 \cdot \sin (2 \cdot \pi \cdot 1 \cdot t)$. For the simulation OrCAD PSpice ver. 16.3 Demo was used.

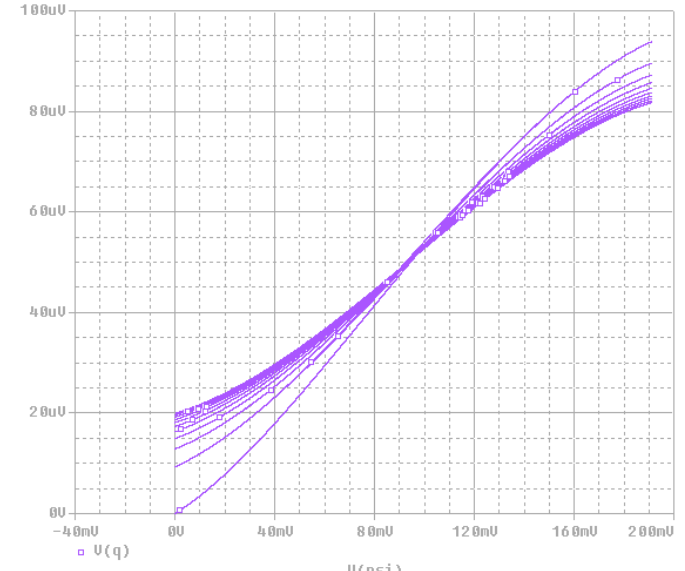

(a)

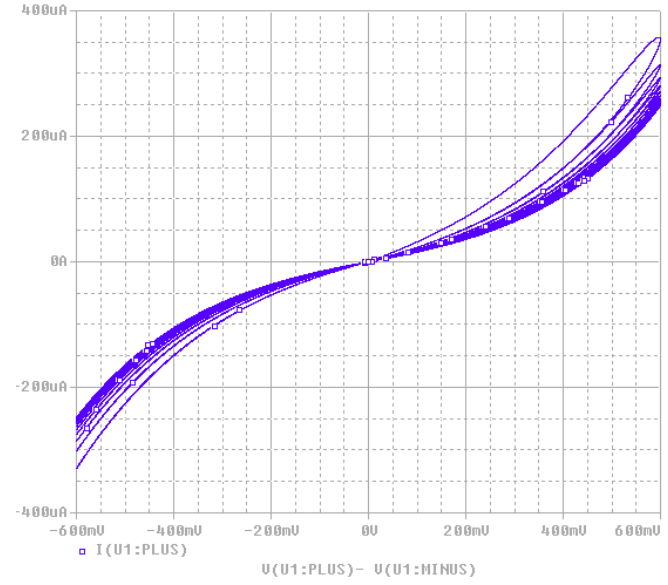

(b)

Figure 3. (a) Weber-Coulomb relationship according to Pickett model; (b) I-V relationship of the memristor element according to Pickett model, $u(t)=0.6 \cdot \sin (2 \cdot \pi \cdot 1 \cdot t)$.

\section{Basic Ideas and the Main Relationships of the Modified Biolek Model}

The modified memristor model proposed here will be discussed using the titanium-dioxide memristor nanostructure described in [2] by Strukov and Williams. A graph of the memristor structure according to the model proposed by Strukov and Williams is presented in Figure 4. The left region of the $\mathrm{TiO}_{2}$ memristor structure with a length of $l$ is partially doped with oxygen vacancies using an initial electroforming process with a high constant voltage and has low resistance [2]. The second sub-layer of the memristor element is prepared of pure $\mathrm{TiO}_{2}$ and it has very high resistance [2]. The length of the whole memristor nanostructure is denoted with $\mathrm{D}$ and has a value of $10 \mathrm{~nm}$ [2]. 


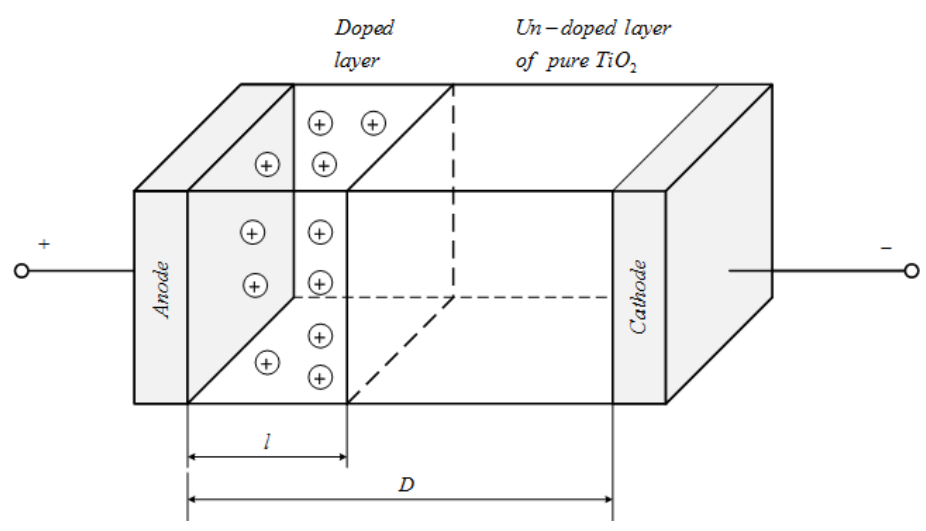

Figure 4. Titanium dioxide memristor nanostructure.

The normalized length of the doped layer, also known as the state variable $x$ of the memristor [2,3], could be defined with the following formula (12):

$$
x=\frac{l}{D}
$$

The equivalent resistance of the memristor element could be expressed using the assumption for series connection of the doped and the un-doped regions [2,3] and the substituting circuit given in Figure 5:

$$
R=R_{\text {doped }}+R_{\text {un-doped }}=R_{O N} x+R_{O F F}(1-x)
$$

where $R_{O N}=100 \Omega$ and $R_{O F F}=16 \mathrm{k} \Omega$ are the memristances of the memristor for fully-closed and fully-open states [2,7], respectively, for $x=1$ or $x=0$.

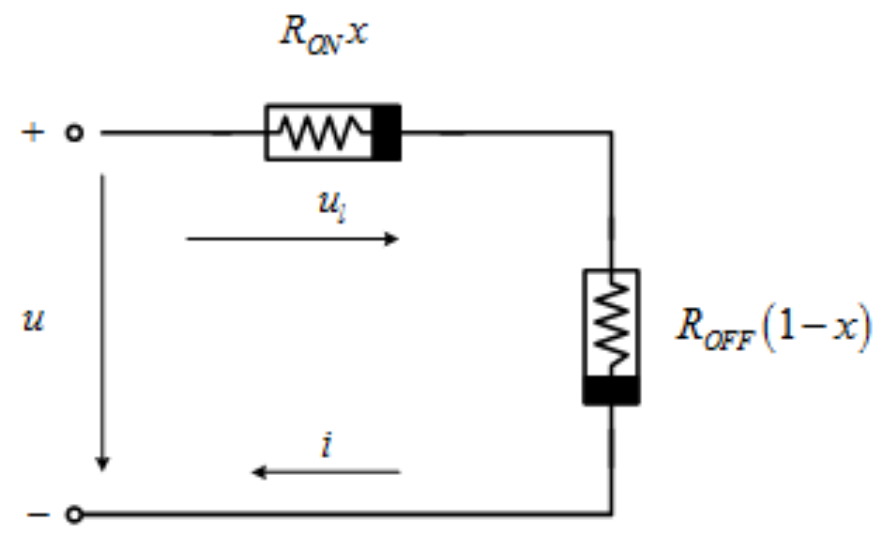

Figure 5. A substituting circuit of the titanium dioxide memristor element presenting the state-dependent resistances of the memristor layers.

The current-voltage relationship could be expressed using (13) and Ohm's law [11]:

$$
u=R i=\left[R_{O N} x+R_{O F F}(1-x)\right] i
$$

The voltage drop across the doped region of the memristor element $u_{l}$ [11] is:

$$
u_{l}=R_{\text {doped }} i=R_{O N} x i=R_{O N} i \frac{l}{D}
$$


The electric field intensity in the doped layer of the memristor element $E_{l}$ is [11,12]:

$$
E_{l}=\frac{u_{l}}{l}=i \frac{R_{O N}}{D}
$$

The rate of moving the boundary between the doped and the un-doped layers of the memristor element [12] is:

$$
v=\frac{d l}{d t}=\frac{d}{d t}(x D)=D \frac{d x}{d t}=\mu E_{l}
$$

where $\mu=1 \cdot 10^{-14} \mathrm{~m}^{2} /(\mathrm{V} \cdot \mathrm{s})$ is the mobility of the oxygen vacancies [2,7]. After transformation of (17) and using (16) the following Equation (18) is derived:

$$
\frac{d x}{d t}=\frac{\mu R_{O N}}{D^{2}} i=k i
$$

where $k$ is a constant dependent only on memristor parameters . Using (18), the maximal amount of electric charges $q_{\max }$ that the memristor accumulates could be calculated [11] when $x=1$ :

$$
q_{\max }=\int_{0}^{\tau} i(t) d t=\frac{1}{k} \int_{0}^{1} d x=\frac{1}{k}=\frac{D^{2}}{\mu R_{O N}}=\frac{\left(10 \cdot 10^{-9}\right)^{2}}{1 \cdot 10^{-14} \cdot 100}=1 \cdot 10^{-4} \mathrm{C}
$$

where the time for charging the memristor $\tau$ depends on the current intensity $i$. Using (18) and (19) it could be derived that the electric charge accumulated in the memristor element is proportional to the maximal amount of electric charge $q_{\max }$ and the state variable $x$ [11]:

$$
q(x)=\int_{0}^{\tau_{1}<\tau} i(t) d t=\frac{1}{k} \int_{0}^{x} d y=q_{\max } x=10^{-4} \cdot x
$$

where in the integral in the right side of (20) the state variable $x$ is substituted with the variable $y$, and $x$ is the upper limit of the definite integral. When two or more memristor elements are connected in an electric circuit $[7,8]$ formula (18) is modified and Equation (21) is derived:

$$
\frac{d x}{d t}=\eta \frac{\mu R_{O N}}{D^{2}} i=\eta k i
$$

where $\eta$ is a polarity coefficient $[7,8]$. When the memristor element is forward-biased then $\eta=1$. For a reverse-biased memristor $\eta=-1[7,8]$. Formula (21) is valid only for very small electrical currents and memristor voltages [2]. For representing the phenomenon of a nonlinear ionic dopant drift in the general case an additional nonlinear window function $f(x)$ has to be used in the right side of Equation $(21)[3,6,7]$ and the following formula is derived (22):

$$
\frac{d x}{d t}=\eta \frac{\mu R_{O N}}{D^{2}} i f(x)=\eta k i f(x)
$$

Equations (14) and (22) fully describe the general memristor model.

Several different basic window functions are used in the memristor modeling [3,6,7].

A very important and frequently used window function is [6]:

$$
f_{B}(x, i)=1-[x-\operatorname{st} p(-i)]^{2 p}
$$

The function expressed with (23) is presented for first use by Biolek in [6], and it is also known as a Biolek window function [6]. The function $\operatorname{st} p(i)$ [6] used in (23) is: 


$$
\operatorname{stp}(i)=\left\{\begin{array}{lll}
1, & \text { if } i \geq 0 & (u \geq 0) \\
0, & \text { if } i<0 & (u<0)
\end{array}\right.
$$

After substitution of (24) [6] in (23) the following equation is derived:

$$
\begin{aligned}
& f_{B}(x)=1-(x-1)^{2 p}, \quad u(t) \leq 0, \quad[i(t) \leq 0] \\
& f_{B}(x)=1-x^{2 p}, \quad u(t)>0, \quad[i(t)>0]
\end{aligned}
$$

The original Biolek model [6] is fully described with Equations (14), (22), and (25).

Here a simple modification of (25) is made. To increase the nonlinearity extent of the modified Biolek model, the authors proposed an additional weighted sinusoidal window function of the state variable $x$ :

$$
\begin{aligned}
& f_{B M}(x)=\left[\frac{1-(x-1)^{2 p}+m\left(\sin ^{2}(\pi x)\right)}{1+m}\right], \quad u(t) \leq 0 \\
& f_{B M}(x)=\left[\frac{1-x^{2 p}+m\left(\sin ^{2}(\pi x)\right)}{1+m}\right], \quad u(t)>0
\end{aligned}
$$

where $m$ is in the interval $[0,1]$ and $f_{B M}$ is the modified window function. The modified Biolek model proposed in this paper is fully described with the following Equation (27):

$$
\begin{aligned}
& \frac{d x}{d t}=\eta k i\left[\frac{1-(x-1)^{2 p}+m\left(\sin ^{2}(\pi x)\right)}{1+m}\right], \quad u(t) \leq 0 \\
& \frac{d x}{d t}=\eta k i\left[\frac{1-x^{2 p}+m\left(\sin ^{2}(\pi x)\right)}{1+m}\right], \quad u(t)>0 \\
& u=R i=\left[R_{O N} x+R_{O F F}(1-x)\right] i
\end{aligned}
$$

If $m=0$ and if the activation threshold of the memristor $u_{t h r}=0$ the modified Biolek model is transformed into the original Biolek model. So it could be concluded that the original Biolek memristor model is a special case of the modified Biolek model proposed in this paper.

\section{A Pseudo-Code Algorithm for Simulation of the Modified Biolek Model}

The pseudo-code algorithm presented below is based on Equation (27) and its numerical solution with the use of the finite differences method in MATLAB [13]. The readers could use this code if they want to simulate memristors.

1. The basic code used for simulations of the modified Biolek model

2. Constants and parameters: $q \max =0.0001 ; \mathrm{eta}=1 ; \mathrm{um}=3.3 ; \mathrm{f}=2.01 ; \mathrm{psiu}=-\mathrm{pi} / 3$; $\mathrm{Ron}=100$;

3. Roff=16000; deltaR=Ron-Roff; $\mathrm{mu}=1 \mathrm{e}-14 ; \mathrm{D}=10 \mathrm{e}-9 ; \mathrm{k}=\left(\mathrm{mu}^{*}\right.$ Ron $) /\left(\mathrm{D}^{\wedge} 2\right) ; \mathrm{x} 0=0.3$;

4. $\quad x \min =0 ; x \max =1 ;[\mathrm{u}, \mathrm{t}$,deltat,tmin,tmax, $\mathrm{N}]=\operatorname{sine} \_g e n(u m, f, p s i u)$;

5. $\mathrm{n}=1: 1: \mathrm{N}+1$; flux=integr(u,deltat,tmin,tmax);

6. [x,biolekwinmod]=biolek_mod(deltat,u,k,eta,deltaR,Roff, $x 0, x \max , \mathrm{xmin}, \mathrm{N})$;

7. $\quad$ Req=deltaR ${ }^{*} \mathrm{x}+$ Roff; $\mathrm{iM}=\mathrm{u} . /$ Req; $\mathrm{q}=\mathrm{qmax}^{*} \mathrm{x}$;

8. Function for generation of sinusoidal voltage signal

9. function $[\mathrm{u}, \mathrm{t}, \mathrm{deltat}, \mathrm{tmin}, \mathrm{tmax}, \mathrm{N}]=$ sine_gen(um,f,psiu)

10. Time domain: $\mathrm{T}=1 / \mathrm{f} ; \mathrm{tmin}=0 ; \mathrm{tmax}=8^{*} \mathrm{~T} ;$ deltat $=(\mathrm{tmax}-\mathrm{tmin}) / 1 \mathrm{e} 5 ; \mathrm{t} 1=\mathrm{tmin}:$ deltat:tmax;

11. omega $=2^{*} \mathrm{pi}^{* \mathrm{f}} ; \mathrm{f} 1=\mathrm{um}^{*} \sin \left(\right.$ omega $\left.{ }^{*} \mathrm{t} 1+\mathrm{psiu}\right) ; \mathrm{u}=\mathrm{f} 1 ; \mathrm{t}=\mathrm{t} 1 ; \mathrm{N}=\mathrm{tmax} /$ deltat; end function.

12. Function for integrating the memristor voltage

13. function $\mathrm{psi}=$ integr(u,deltat,tmin,tmax)

14. $\mathrm{N}=($ tmax-tmin)/deltat; $\mathrm{n}=1: 1: \mathrm{N}+1 ; \mathrm{psi}=[\mathrm{]}$;

15. for $\mathrm{n}=1 ; \mathrm{psi} 1(\mathrm{n})=0$; end;

16. for $n=2: 1: N+1 ; \operatorname{psi} 1(n)=p s i 1(n-1)+u(n-1)^{*}$ deltat; end; 
17. psi=[psi psi1]; end function.

18. Function for deriving the state variable and the modified Biolek window

19. function $[\mathrm{x}$, biolekwinmod] $=$ biolek_mod(deltat,u,k,eta,deltaR,Roff, $x 0, \mathrm{xmax}, \mathrm{xmin}, \mathrm{N})$

20. Parameters: $\mathrm{p}=2 ; \mathrm{m}=0.2 ; \mathrm{x}=[\mathrm{]}$; biolekwinmod $=[\mathrm{]}$; for $\mathrm{n}=1 ; \mathrm{x} 1=\mathrm{x} 0$; biolekmod $1=1$; end;

21. for $n=2: 1: N+1 ; A=\operatorname{deltaR}^{*} x 1(n-1)+\operatorname{Roff} ;$ if $\operatorname{abs}(u(n))<0.1$; then $x 1(n)=x 1(n-1)$;

22. if $\mathrm{u}(\mathrm{n})<=0$; then biolekmod $1(\mathrm{n})=\left(\left(\left(1-(\mathrm{x} 1(\mathrm{n})-1)^{\wedge}\left(2^{*} \mathrm{p}\right)\right)+\mathrm{m}^{*}\left(\sin \left(\mathrm{pi}^{*} \mathrm{x} 1(\mathrm{n})\right)\right)^{\wedge} 2\right)\right) /(1+\mathrm{m})$;

23. else biolekmod1 $(\mathrm{n})=\left(\left(\left(1-(x 1(\mathrm{n}))^{\wedge}\left(2^{*} \mathrm{p}\right)\right)+\mathrm{m}^{*}\left(\sin \left(\mathrm{pi}^{*} \mathrm{x} 1(\mathrm{n})\right)\right)^{\wedge} 2\right)\right) /(1+\mathrm{m})$; end;

24. else; if $\mathrm{u}(\mathrm{n})<=0$; then $\mathrm{x} 1(\mathrm{n})=\mathrm{x} 1(\mathrm{n}-1)+\left(\operatorname{eta}^{*} \mathrm{k}^{*} \mathrm{u}(\mathrm{n}-1)^{*} \operatorname{deltat}^{*}(1 / \mathrm{A})^{*}\left(\left(\left(1-(\mathrm{x} 1(\mathrm{n}-1)-1)^{\wedge}\left(2^{*} \mathrm{p}\right)\right)+\ldots\right.\right.\right.$

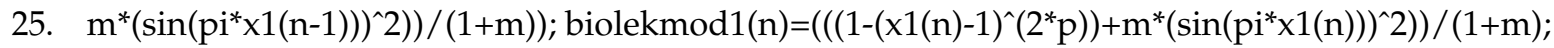

26. if $x 1(n)<=x \min \& \mathrm{u}<=0$; then $x 1(n)=x \min$; else; $x 1(n)=x 1(n-1)+\left(\operatorname{eta}^{*} k^{*} u(n-1)^{*} \operatorname{deltat}^{*}(1 / A)^{*}(((1-(x 1\right.$ $(n-1)-1) \ldots$

27. $\left.\left.\left.\left.\wedge^{\wedge}\left(2^{*} \mathrm{p}\right)\right)+\mathrm{m}^{*}\left(\sin \left(\mathrm{pi}^{*} \mathrm{x} 1(\mathrm{n}-1)\right)\right)^{\wedge} 2\right)\right) /(1+\mathrm{m})\right)$; $\operatorname{biolekmod} 1(\mathrm{n})=\left(\left(\left(1-(\mathrm{x} 1(\mathrm{n})-1)^{\wedge}\left(2^{*} \mathrm{p}\right)\right)+\mathrm{m}^{*}\left(\sin \left(\mathrm{pi}^{*} \mathrm{x} 1(\mathrm{n})\right)\right)^{\wedge} 2\right)\right) /$ $(1+\mathrm{m})$;

28. end; else $x 1(n)=x 1(n-1)+\left(\operatorname{eta}^{*} k^{*} u(n-1)^{*} \operatorname{deltat}^{*}(1 / A)^{*}\left(\left(\left(1-(x 1(n-1))^{\wedge}\left(2^{*} p\right)\right)+m^{*}\left(\sin \left(\operatorname{pi}^{*} x 1(n-1)\right)\right)^{\wedge} 2\right)\right) /\right.$ $(1+\mathrm{m}))$;

29. $\operatorname{biolekmod} 1(n)=\left(\left(\left(1-(x 1(n))^{\wedge}\left(2^{*} \mathrm{p}\right)\right)+m^{*}\left(\sin \left(\mathrm{pi}^{*} \mathrm{x} 1(\mathrm{n})\right)\right)^{\wedge} 2\right)\right) /(1+\mathrm{m}) ; \quad$ if $\mathrm{x} 1(\mathrm{n})>=x \max \quad \& \quad \mathrm{u}(\mathrm{n})>0$; $x 1(n)=x m a x ;$

30. else $x 1(n)=x 1(n-1)+\left(\operatorname{eta}^{*} k^{*} u(n-1)^{*} \operatorname{deltat}^{*}(1 / A)^{*}\left(\left(\left(1-(x 1(n-1))^{\wedge}\left(2^{*} p\right)\right)+m^{*}\left(\sin \left(p^{*} x 1(n-1)\right)\right)^{\wedge} 2\right)\right) /(1+m)\right)$;

31. biolekmod $1(\mathrm{n})=\left(\left(\left(1-(\mathrm{x} 1(\mathrm{n}))^{\wedge}\left(2^{*} \mathrm{p}\right)\right)+\mathrm{m}^{*}\left(\sin \left(\mathrm{pi}^{*} \mathrm{x} 1(\mathrm{n})\right)\right)^{\wedge} 2\right)\right) /(1+\mathrm{m})$; end;

32. end; end; end; $x=[x \times 1]$; biolekwinmod=[biolekwinmod biolekmod1]; end function.

\section{A Comparison with the Pickett Memristor Model, Adjusting of the Modified Biolek Model and the Basic Results Obtained by the Computer Simulations}

\subsection{Adjusting the Modified Memristor Model Using Comparison with the Pickett Model}

Using the pseudo-code presented above for different values of the exponent $p$ and of the coefficient $m$, discussed in the previous section, several simulations were made in MATLAB R2010b environment [13]. After adjusting the coefficients, the basic results were derived. The Weber-coulomb relation shown in Figure 6a is almost similar to those of the Pickett model-Figure 3a. The respective current-voltage relationship is presented in Figure $6 \mathrm{~b}$ and it is almost identical to those of the Pickett model given in Figure $3 \mathrm{~b}$. The adjusted values of the coefficients are $p=7$ and $m=0.2$.

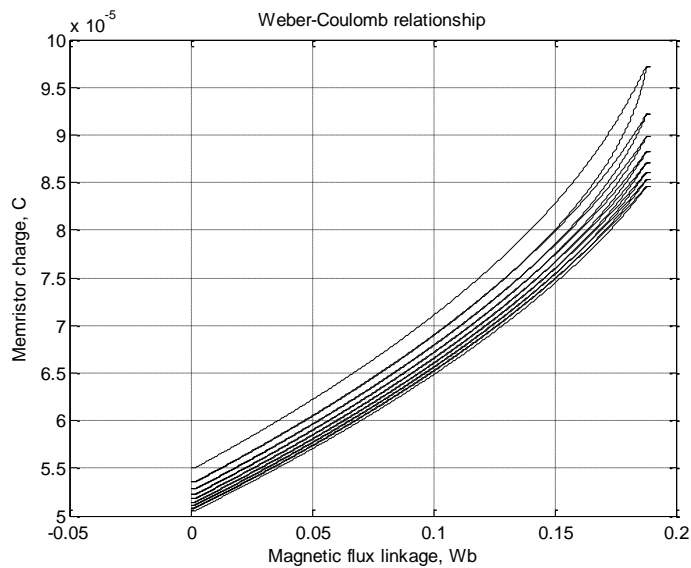

(a)

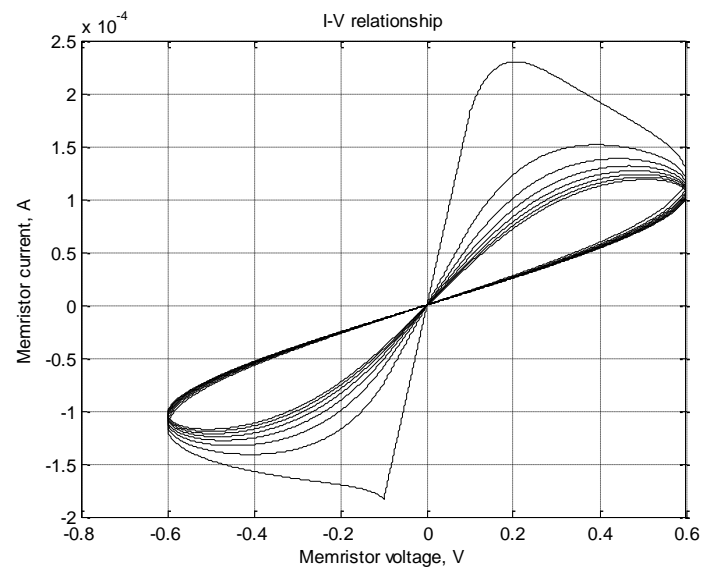

(b)

Figure 6. (a) Weber-Coulomb relationship according to the modified Biolek model; (b) I-V relationship of the memristor element according to the modified Biolek model, $u(t)=0.6 \cdot \sin (2 \cdot \pi \cdot 1 \cdot t)$. 
The respective time diagrams of the memristor voltage and state variable are presented in Figure 7a. The state variable $x$ does not reach its boundary values so it could be concluded that the memristor operates in a soft-switching mode. The relationship between the modified window function and the state variable is presented in Figure $7 \mathrm{~b}$. The operating point of the memristor in the field of the coordinate system moves between two branches of the window function in accordance with the sign of the memristor voltage.

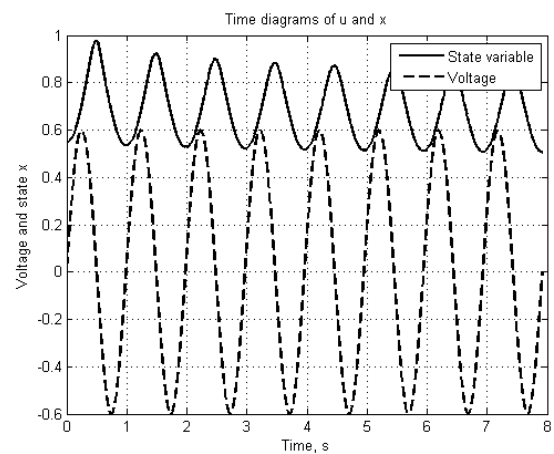

(a)

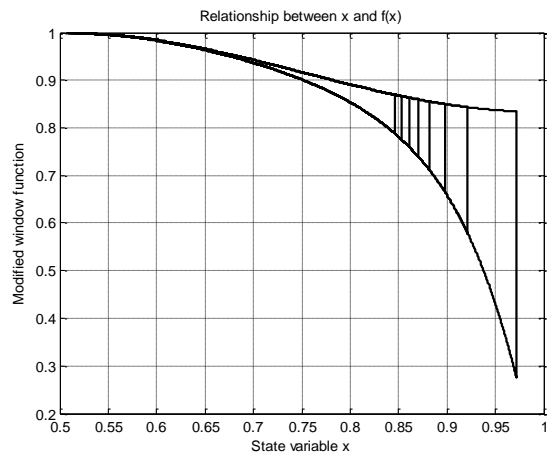

(b)

Figure 7. (a) Time diagrams of memristor voltage and state variable according to the modified Biolek model; (b) The relationship between the modified window function and the state variable of the memristor according to the modified Biolek model, $u(t)=0.6 \cdot \sin (2 \cdot \pi \cdot 1 \cdot t)$.

\subsection{Testing the New Model for Hard-Switching Mode}

The adjusted memristor model was tested for a voltage signal with several times higher amplitude and with negative initial voltage phase $-u(t)=3.6 \cdot \sin \left(2 \cdot \pi \cdot 1 \cdot t-120^{\circ}\right)$. The respective Weber-Coulomb relationship is presented in Figure 8a. In this case it is a multi-valued hysteresis curve which reaches the boundary values of the accumulated in the memristor charge. Then it could be concluded that the memristor operates in a hard-switching mode. The respective current-voltage relationship is presented in Figure $8 \mathrm{~b}$. In this case, the resistance of the memristor for forward biasing is low and the respective resistance for negative voltages is very high. So it could be said that in hard-switching mode the memristor behaves like a rectifying diode. The time diagrams of the memristor voltage and state variable for hard-switching are presented in Figure 9a. In this case, the state variable reaches its limiting values -0 and 1 . The respective relationship between the modified window function and the state variable is shown in Figure $9 \mathrm{~b}$. In this case, the window function values are in the interval between 0 and 1 . The operating point of the memristor in the field of the coordinate system moves on all the length of the two branches in accordance to the memristor voltage sign.

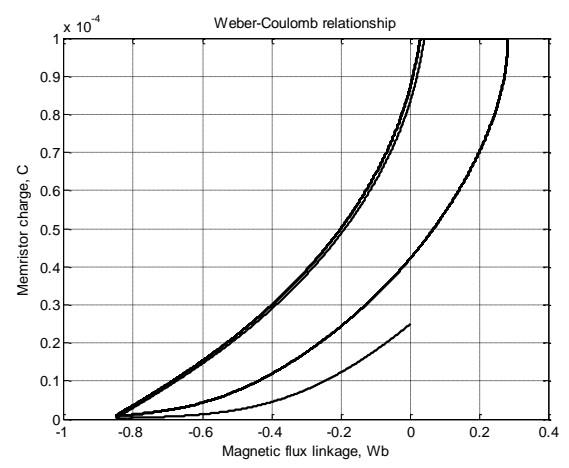

(a)

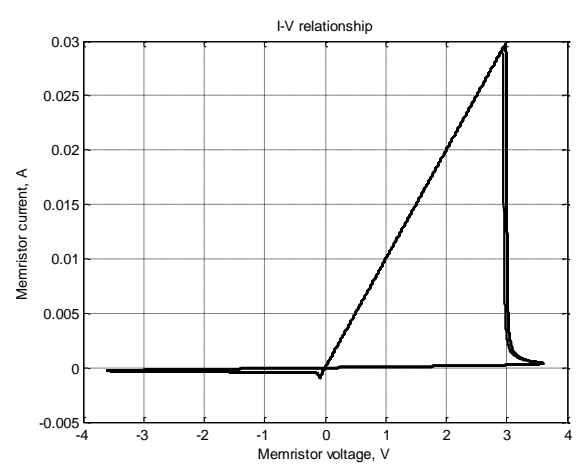

(b)

Figure 8. (a) Weber-Coulomb relationship according to the modified Biolek model; (b) I-V relationship of the memristor element according to the modified Biolek model, $u(t)=3.6 \cdot \sin \left(2 \cdot \pi \cdot 1 \cdot t-120^{\circ}\right)$. 


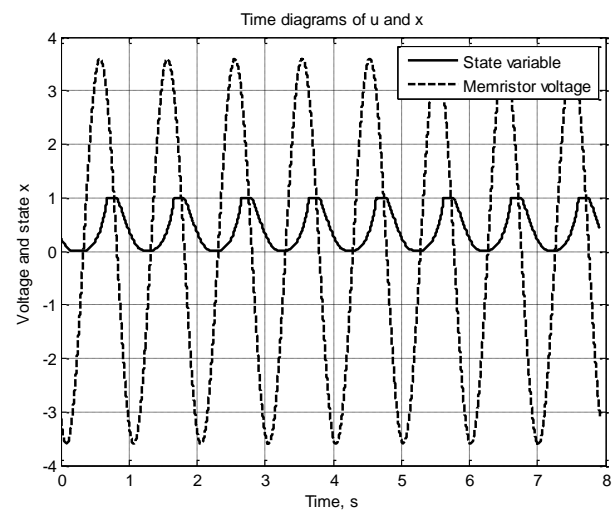

(a)

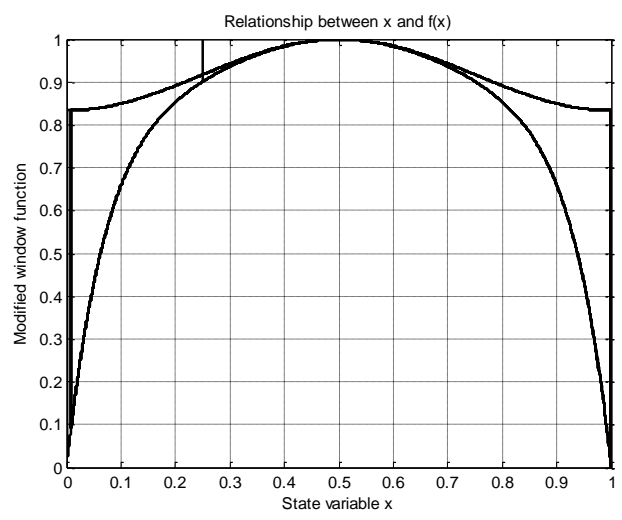

(b)

Figure 9. (a) Time diagrams of memristor voltage and state variable according to the modified Biolek model; (b) The relationship between the modified window function and the state variable of the memristor according to the modified Biolek model, $u(t)=3.6 \cdot \sin \left(2 \cdot \pi \cdot 1 \cdot t-120^{\circ}\right)$.

The test of the modified Biolek model for a pseudo-sinusoidal voltage signal with exponentially increasing amplitude confirms the expected behavior of the model for voltages lower and higher than the activation threshold $u_{t h r}$. The respective Weber-Coulomb relationship in this case is presented in Figure 10a. In the beginning, the operation point of the memristor in the field of the coordinate system moves on a straight line because the state variable does not change for voltages lower that the sensitivity threshold. When the voltage is higher than the activation threshold, the operating point starts to move on a different branch of the Weber-coulomb relationship, which is a curve line, and the state variable changes its value in accordance with the flux linkage. The respective current-voltage relationship is shown in Figure 10b. In the beginning, it is a straight line and for voltages higher than the sensitivity threshold, it is a multi-valued pinched hysteresis loop.

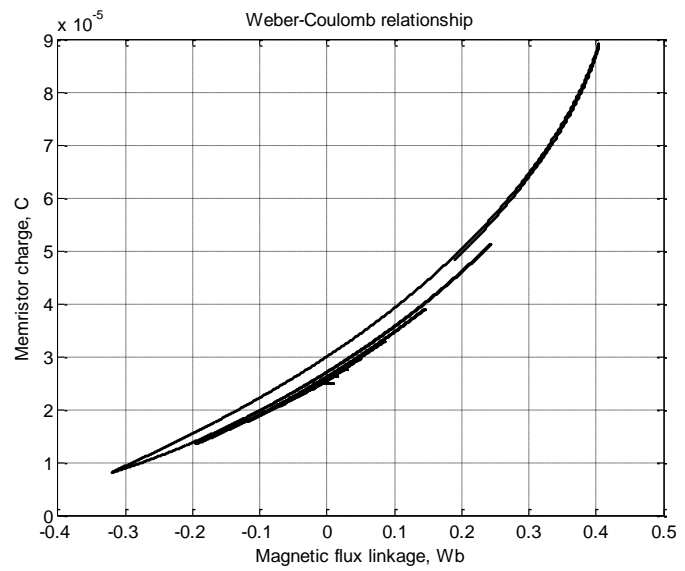

(a)

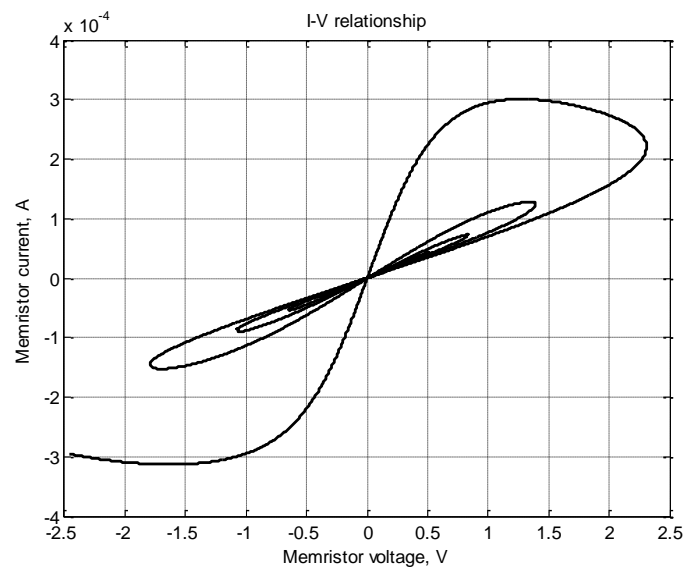

(b)

Figure 10. (a) Weber-Coulomb relationship according to the modified Biolek model; (b) I-V relationship of the memristor element according to the modified Biolek model, $u(t)=0.05 \cdot \exp (0.51 \cdot t) \cdot \sin \left(2 \cdot \pi \cdot 1 \cdot t-120^{\circ}\right)$.

The respective time diagrams of the memristor voltage and the state variable are presented in Figure 11a. The voltage signal is pseudo-sinusoidal with exponentially increasing amplitude. In the beginning for voltages lower than the sensitivity threshold the state variable does not change its value but for higher voltages it starts to change. For voltages lower than the activation threshold, the memristor behaves like a linear resistor. If the signal becomes higher than the activation threshold 
then the state variable changes and the element behaves like a memristor. The relationship between the modified window function and the state variable is presented in Figure 11b.

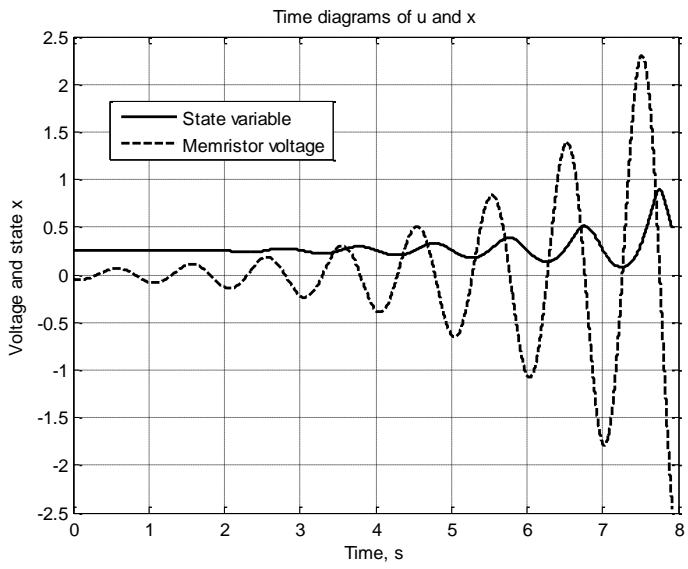

(a)

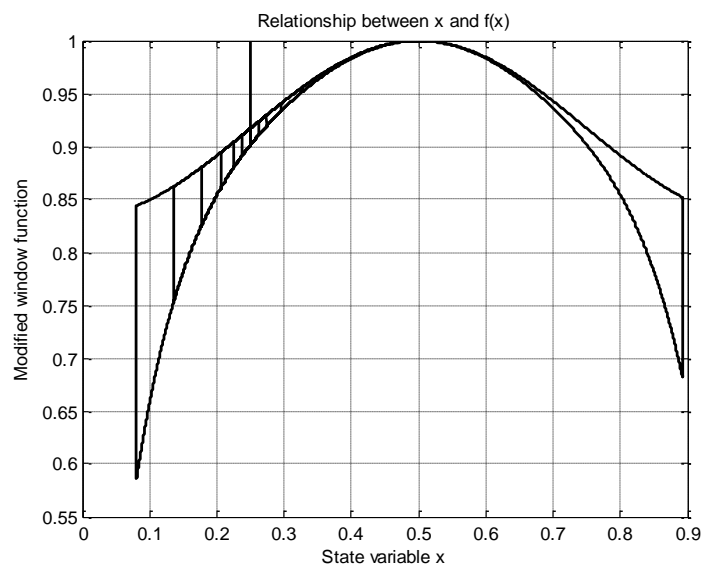

(b)

Figure 11. (a) Time diagrams of memristor voltage and state variable according to the modified Biolek model; (b) The relationship between the modified window function and the state variable of the memristor according to the modified Biolek model, $u(t)=0.05 \cdot \exp (0.51 \cdot t) \cdot \sin \left(2 \cdot \pi \cdot 1 \cdot t-120^{\circ}\right)$.

According to the voltage sign, the operating point of the memristor in the field of the coordinate system moves between the two branches of the characteristics.

\section{A Comparison of the Modified Memristor Model with Existing Models}

In this section a comparison of the modified Biolek model with the Joglekar memristor model [3] for soft-switching mode will be done. According to [3] the window function used in Equation (22) is:

$$
f_{J}(x)=1-(2 x-1)^{2 p}
$$

Equation (28) is presented for first use by Joglekar in [3] and it is also known as Joglekar window function [3]. Using (14), (22), and (28) the Joglekar memristor model could be fully described with Equation (29) where the state-dependent Ohm's Law is also included [3].

$$
\begin{aligned}
& \frac{d x}{d t}=\eta k i\left[1-(2 x-1)^{2 p}\right] \\
& u=R i=\left[R_{O N} x+R_{O F F}(1-x)\right] i
\end{aligned}
$$

The Joglekar memristor model could be simulated using the code given in Section 4 but Equation (28) has to be used instead of the Biolek window function in row 6 in the code. The code of the function for calculating the state variable and the Joglekar window according to the Joglekar memristor model is presented below and it could be used by the readers for simulations [13]:

1. A function for simulating the Joglekar memristor model

2. function $[\mathrm{x}, \mathrm{jog}$ lwin $]=\mathrm{jog} \mathrm{l}($ deltat, $\mathrm{u}, \mathrm{k}, \mathrm{eta}, \mathrm{deltaR}, \mathrm{Roff}, \mathrm{x} 0, \mathrm{~N})$

3. Parameter $\mathrm{p}=2 ; \mathrm{x}=[\mathrm{]}$;joglwin $=[\mathrm{]}$;

4. for $\mathrm{n}=1 ; \mathrm{x} 1=\mathrm{x} 0$; joglwin $1=1-\left(\left(2^{*} \mathrm{x} 1-1\right)^{\wedge}\left(2^{*} \mathrm{p}\right)\right)$; end;

5. for $n=2: 1: N+1 ; x 1(n)=x 1(n-1)+e^{*}{ }^{*} k^{*} \operatorname{deltat}^{*} \mathrm{u}(\mathrm{n}-1)^{*}\left(\left(1-\left(2^{*} x 1(n-1)-1\right)^{\wedge}\left(2^{*} \mathrm{p}\right)\right) /(\operatorname{deltaR} \times 1(n-1)+\operatorname{Roff})\right)$; $\operatorname{jog} \operatorname{lwin} 1(\mathrm{n})=1-\left(\left(2^{*} \times 1(\mathrm{n})-1\right)^{\wedge}\left(2^{*} \mathrm{p}\right)\right)$; end;

6. $\mathrm{x}=[\mathrm{x} \times 1]$; joglwin=[joglwin joglwin1]; end function.

The voltage signal used for simulation of the Joglekar model and the modified model is: $u(t)=$ $3.3 \cdot \sin \left(2 \cdot \pi \cdot 2 \cdot t-60^{\circ}\right)$. The I-V relationships of the memristor are presented in Figure 12a. In this case, 
the I-V curve according to Joglekar's model is double-valued while the modified model represents multi-valued curve. The state-flux relationships are given in Figure 12b. According to Joglekar's model, this relation is single-valued, which is an advantage with respect to the multi-valued state-flux characteristics obtained by the modified model.
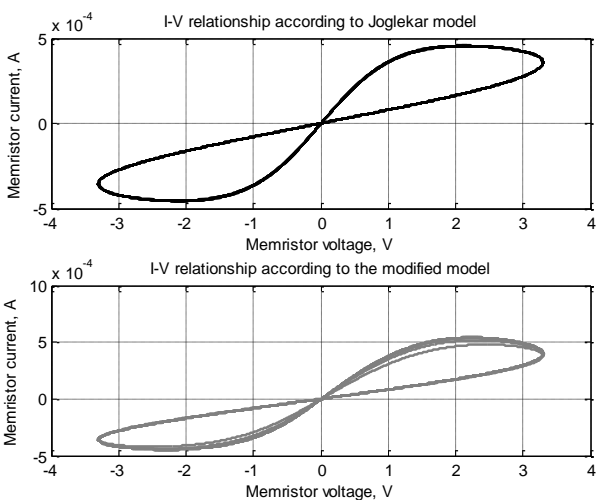

(a)
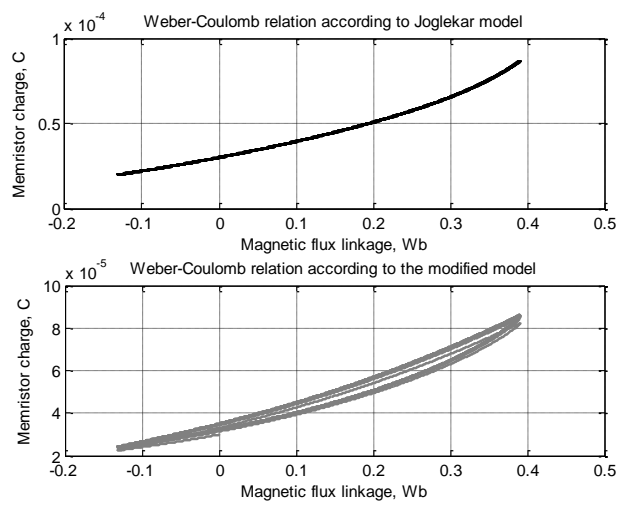

(b)

Figure 12. (a) Current-voltage relations of the memristor according to Joglekar model and the modified model; (b) State-flux relation according to Joglekar's and the modified model, $u(t)=3.3 \cdot \sin \left(2 \cdot \pi \cdot 2 \cdot t-60^{\circ}\right)$.

The time diagrams of the Joglekar memristor model and of the modified model presented in Figure 13a are almost identical. The relationships between the window function values and the state variable $x$ for the Joglekar model, and for the modified memristor model are presented in Figure 13b. The state variable of the memristor models changes in equivalent intervals. The modified Biolek window function in this case provides higher nonlinearity of the memristor, which is an advantage of the modified model with respect to the Joglekar model. Another advantages of the modified Biolek model proposed here is the ability for realistic representation of the boundary effects when the memristor operates in a hard-switching mode and the use of sensitivity threshold. The original Joglekar memristor model does not have mechanism for representing the boundary effects and it is appropriate only for simulating of memristors for soft-switching mode.
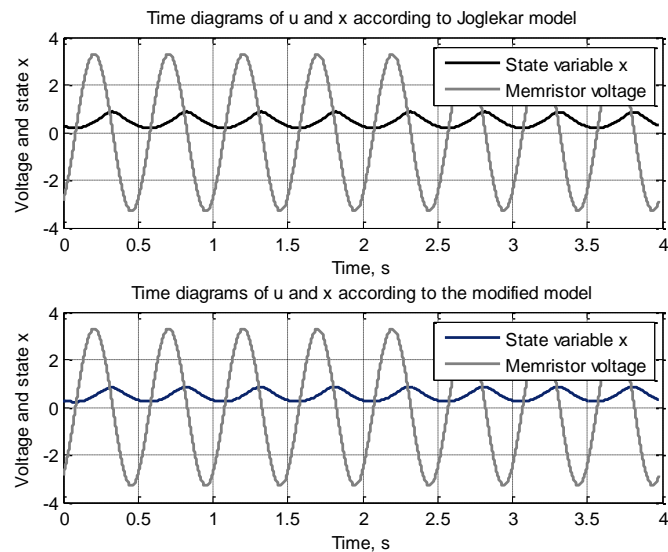

(a)
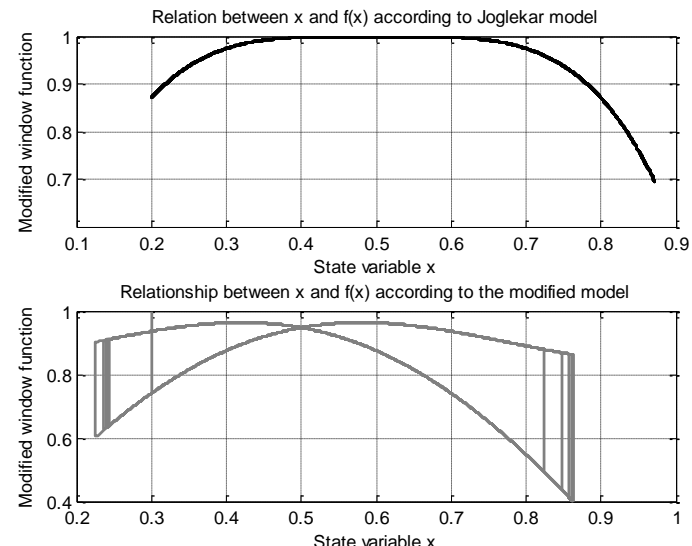

(b)

Figure 13. (a) Time diagrams of the memristor voltage and state variable according to Joglekar model and the modified memristor model; (b) Relationship between the window function value and the state variable according to Joglekar memristor model and the modified Biolek model, $u(t)=3.3 \cdot \sin \left(2 \cdot \pi \cdot 2 \cdot t-60^{\circ}\right)$. 


\section{Conclusions}

After finishing the adjusting process of the modified Biolek model and obtaining the basic results, several conclusions could be made. The modified memristor model based both on Biolek model and GBCM model is a general one and it contains a modified window function, which is a sum of the original Biolek window function, and a weighted sinusoidal window function. In the special case when the coefficient in front of the sinusoidal window function and the activation threshold are chosen to be equal to zero, the original Biolek memristor model is obtained. If the modified model presented here is adjusted, we could obtain results identical to those obtained by the Pickett model. Of course, the results obtained in the present research are not exactly the same like those produced by Pickett model, which has the highest accuracy but also has many convergence problems and is not appropriate for simulations. The main advantage of the modified Biolek model with respect to the Pickett model is the lack of convergence problems and the possibility for use of the modified model for computer simulations of memristors and memristive circuits.

The modified memristor model proposed here was compared with Joglekar memristor model for soft-switching mode. The state-flux relationship obtained by Joglekar model is a single-valued curve, which is an advantage of Joglekar model with respect to the modified model which state-flux characteristics are multi-valued. On the other hand, the modified memristor model proposed in this paper has several advantages with respect to Joglekar memristor model—higher nonlinearity of the dopant drift, ability for realistic representation of the boundary effects, and the use of sensitivity voltage threshold. After comparison of the modified memristor model proposed in this paper with the Pickett model it could be concluded that the Pickett model could be simulated for a narrow range of voltages and for higher voltages convergence problems occur. During the tests of Pickett model only operation in a soft-switching mode was observed. An advantage of the model proposed in this research with respect to the Pickett model is the possibility for simulating the modified model in a broaden voltage range and the observation of hard-switching mode behavior.

Acknowledgments: The research is supported by national Co-financing (contract № ДKOCT01/14) of COST Action № IC1401 MemoCIS.

Author Contributions: V.M. and S.K. made the modified computer memristor model and simulations; V.M. analyzed the results and adjusted the new memristor model in accordance with the Pickett model; V.M. and S.K. compared the modified memristor model with Joglekar model and prepared the manuscript of the present paper.

Conflicts of Interest: The authors declare no conflict of interest.

The founding sponsors had no role in the design of the study; in the collection, analyses, or interpretation of data; in the writing of the manuscript, and in the decision to publish the results.

\section{References}

1. Chua, L. Memristor-The Missing Circuit Element. IEEE Trans. Circuit Theory 1971, 18, 507-519. [CrossRef]

2. Strukov, D.; Snider, G.; Stewart, D.; Williams, R.S. The Missing Memristor Found. Nat. Lett. 2008, 453, 80-83. [CrossRef] [PubMed]

3. Joglekar, Y.; Wolf, S. The Elusive Memristor: Properties of Basic Electrical Circuits. Eur. J. Phys. 2009, 30, 661-675. [CrossRef]

4. Pickett, M.; Strukov, D.; Borghetti, J.; Yang, J.; Snider, G.; Stewart, D.; Williams, R.S. Switching dynamics in titanium dioxide memristive devices. J. Appl. Phys. 2009, 106, 1-6. [CrossRef]

5. Abdalla, H.; Pickett, M. SPICE modeling of memristors. IEEE Int. Symp. Circuits Syst. 2011, 1832-1835. [CrossRef]

6. Biolek, Z.; Biolek, D.; Biolkova, V. SPICE Model of Memristor with Nonlinear Dopant Drift. Radioengineering 2009, 18, 210-214.

7. Corinto, F.; Ascoli, A. A Boundary Condition-Based Approach to the Modeling of Memristor Nanostructures. IEEE Trans. Circuits Syst.I Regul. Pap. 2012, 59, 2713-2726. [CrossRef] 
8. Ascoli, A.; Corinto, F.; Tetzlaff, R. Generalized Boundary Condition Memristor Model. Int. J. Circuit Theory Appl. 2016, 44, 60-84. [CrossRef]

9. Ascoli, A.; Corinto, F.; Senger, V.; Tetzlaff, R. Memristor Model Comparison. IEEE Circuits Syst. Mag. 2013, 13, 89-105. [CrossRef]

10. Ascoli, A.; Tetzlaff, R.; Biolek, Z.; Kolka, Z.; Biolkovà, V.; Biolek, D. The Art of Finding Accurate Memristor Model Solutions. IEEE J. Emerg. Sel. Top. Circuits Syst. 2015, 5, 133-142. [CrossRef]

11. Brandisky, K.; Georgiev, Z.; Mladenov, V.; Stancheva, R. Theoretical Electrical Engineering-Part 1 E 2; KING Publishing House: Sofia, Bulgaria, 2005; ISBN 954-9518-28-0. (In Bulgarian)

12. Hristov, M.; Vassileva, T.; Manolov, E. Semiconductor Elements; New Knowledge Publishing House: Sofia, Bulgaria, 2007; ISBN 978-954-9315-79-0. (In Bulgarian)

13. Tonchev, Y. MATLAB 7 - transformations, calculations, visualization - Part 1; Technical Publishing House: Bulgaria, 2010; ISBN 978-954-03-0694-0. (In Bulgarian)

(C) 2017 by the authors. Licensee MDPI, Basel, Switzerland. This article is an open access article distributed under the terms and conditions of the Creative Commons Attribution (CC BY) license (http:/ / creativecommons.org/licenses/by/4.0/). 